\title{
Alterações renais clínico-laboratoriais em pacientes com a síndrome da imunodeficiência adquirida em relação aos achados anatomopatológicos
}

\author{
Clinical and laboratory renal alterations in patients with acquired \\ immunodeficiency syndrome in relation to anatomopathological findings \\ Victor Alberto Laguna-Torres, Marlene Antônia dos Reis, Dalmo Correia, \\ Mário León Silva-Vergara e Aluízio Prata
}

\begin{abstract}
Resumo Foram estudadas as alterações renais, tanto clínicas como anatomopatológicas, em 119 indivíduos com a síndrome da imunodeficiência adquirida no Hospital Escola da Faculdade de Medicina do Triângulo Mineiro, Uberaba, MG. A média das idades foi de $33 \pm 10,4$ anos (variando de 18 a 67 anos). Predominaram a cor branca (59\%) e o sexo masculino (80\%). Procedência: $90 \%$ de Uberaba e região. Havia 59 (50\%) heterossexuais, 25 (21\%) homossexuais, $17(14 \%)$ bissexuais e $18(15 \%)$ sem dados conhecidos. Havia $43 \%$ usuários de drogas intravenosas. Catorze (12,2\%) casos apresentavam creatininemia acima de $3 \mathrm{mg} / \mathrm{dl}$ nos 10 dias antes do óbito, destes encontrou-se necrose tubular aguda em 64\%. Em 71 (61\%) pacientes encontrou-se hiponatremia e 8 (7\%) exibiram hipernatremia. Houve 28 (24\%) com hipocalemia e 8 (7\%) com hipercalemia. Doze pacientes tinham proteinúria de 24 horas elevada, sendo 7 acima de 1g/24h. Dois pacientes apresentaram glomeruloesclerose segmentar focal, sendo que um deles apresentou proteinúria de $5,5 \mathrm{~g} / 24 \mathrm{~h}$ e creatinemia de 1,5mg/dl, sem edema. Na maioria (56\%) dos casos, encontrou-se nefrite túbulointersticial. As alterações glomerulares foram relativamente raras em tanto que as lesões túbulointersticiais foram muito freqüentes, principalmente a nefrite túbulo intersticial e a necrose tubular aguda. Nem sempre se pode observar uma correlação anatomoclínica pois, muitas vezes, a alteração foi predominantemente funcional, especialmente nas hidroeletrolíticas.
\end{abstract}

Palavras-chaves: VIH. SIDA. Necrose tubular aguda. Hiponatremia. Nefrite túbulo-intersticial .

\begin{abstract}
Clinical as well as renal anatomopathological data of 119 patients with acquired immunodeffiency syndrome from the Hospital of the Faculty of Medicine of Triângulo Mineiro, Uberaba, MG, Brazil, were studied. Mean age was $33 \pm 10.4$ years (range 18 to 67 years). White (59\%) and male (80\%) individuals predominated. Ninety percent of patients came from Uberaba or neighborhood. There were 59 (50\%) heterosexual, 25 (21\%) homosexual, 17 (14\%) bisexual and 18 (15\%) unknown. Forty three percent were drug abusers. Fourteen $(12.2 \%)$ of patients had serum creatinine higher than $3 \mathrm{mg} / \mathrm{dl}$, ten days before death, $64 \%$ of them showed acute tubular necrosis. Seventy one (61\%) patients presented hyponatremia and $8(7 \%)$ had hypernatremia. Hypokalemia was detected in $28(24 \%)$ and hyperkalemia in $8(7 \%)$ patients. Twenty four hours urinary protein was detected in 12 patients, 7 of them presenting more than $1 \mathrm{~g} / 24 \mathrm{~h}$. Two patients showed segmentar focal glomerulosclerosis, one of them with $24 \mathrm{~h}$ urinary protein level of $5.5 \mathrm{~g}$ and serum creatinine $1.5 \mathrm{mg} / \mathrm{dl}$ and no edema. The most (56\%) patients had tubulointerstitial nephritis. Clinical, laboratory and types of renal disorders in patients with acquired immunodeffiiency syndrome are variable. The glomerular diseases were relatively scarce however, tubulointerstitial lesions are quite frequent, mainly interstitial nephritis and acute tubular necrosis. Not always an anatomoclinical correlation could be observed, because many times the electrolyte disorders were frequentely observed only functionaly.
\end{abstract}

Key-words: HIV. AIDS. Acute tubular necrosis. Hyponatremia. Tubulointerstitial nephritis.

Disciplinas de Doenças Infecciosas e Parasitárias e Patologia Geral da Faculdade de Medicina do Triângulo Mineiro, Uberaba, MG Endereço para correspondência: Dr.Victor Alberto Laguna-Torres. Los Amancaes, 381, URB, Jardines Virú, Callao 2, Lima, Perú. Fax: (0051) 14775650.

e-mail: alaguna@oge.sld.pe

Recebido para publicação em 19/1/99. 
Há mais de uma década, a glomeruloesclerose segmentar focal (GESF) foi descrita como a nefropatia mais freqüentemente associada ao vírus da imunodeficiência humana $(\mathrm{VIH})^{92536}$. Os pacientes com síndrome de imunodeficiência adquirida (SIDA) podem também desenvolver outras alterações renais, incluindo nefrite túbulointersticiais (NTI), associada ou não a agentes infecciosos, necrose tubular aguda (NTA) e glomerulopatias diversas ${ }^{79262736}$.

A insuficiência renal é uma complicação freqüentemente observada entre os pacientes com SIDA, podendo ser secundária à isquemia devido a distúrbio hemodinâmico, sepses e, inclusive, ao uso de drogas nefrotóxicas ${ }^{2635}$.

Dentre as alterações hidroeletrolíticas, destacam-se a hiponatremia, em 40 a $52 \%$ dos casos $^{1022}{ }^{24}$ e hipernatremia, em 24 a $75 \%$ dos pacientes hospitalizados ${ }^{12}$. Os distúrbios do potássio, são menos freqüentes e podem aparecer como conseqüência das perdas gastrointestinais, devido às drogas, utilizadas na terapêutica ${ }^{2}$, ou à insuficiência supra-renal ${ }^{12} 13$.

O objetivo deste estudo é descrever as manifestações clínicas e os achados laboratoriais renais, correlacionando-os com os dados anatomopatológicos já publicados ${ }^{18}$.

\section{MATERIAL E MÉTODOS}

O estudo foi realizado no Hospital Escola da Faculdade de Medicina do Triângulo Mineiro (HE/ FMTM), Uberaba, Minas Gerais. Os doentes incluídos nesta amostra foram atendidos entre 1989 e fevereiro de 1997.

Em 119 casos estudados, foram coletados os seguintes dados dos prontuários dos indivíduos: nome, idade, cor, sexo, procedência, conduta sexual e informações sobre o uso de drogas intravenosas. As características clínico-laboratoriais estudadas foram: tensão arterial, edema de membros inferiores, níveis de creatinina e eletrólitos séricos. Foram verificadas, também, a proteinúria de 24 horas e a depuração da creatinina endógena.

A dosagem de creatinina sérica foi determinada colorimetricamente, utilizando-se o método de Jaffé ${ }^{33}$. Considerou-se como normais os valores de $0,4-1,2 \mathrm{mg} / \mathrm{dl}$. Foram padronizadas as seguintes categorias: azotemia, quando o nível de creatinina sangüínea estava entre $1,2 \mathrm{mg} / \mathrm{dl}$ e $3 \mathrm{mg} / \mathrm{dl}$ e insuficiência renal estabelecida (IRE), quando acima de $3 \mathrm{mg} / \mathrm{dl}$. Este último valor foi considerado por ter maior chance de uma correlação anatomopatológica renal ${ }^{5}$.

Estabeleceu-se como normonatremia os valores entre $135-150 \mathrm{mEq} / \mathrm{l}$. A hiponatremia quando inferior a $120 \mathrm{mEq} / \mathrm{l}$ foi considerada grave. Os valores da potassemia entre 3 e $5,5 \mathrm{mEq} / \mathrm{l}$ foram considerados normais. O limite dos valores normais para proteínas na urina de $24 \mathrm{~h}$ foi até $150 \mathrm{mg}$. A proteinúria acima de $3,5 \mathrm{~g}$ foi considerada de nível nefrótico ${ }^{16}$.

Quanto aos valores de depuração de creatinina, considerou-se normais quando estavam entre
100 e $140 \mathrm{ml} / \mathrm{min}$, em homens adultos para uma superfície corporal de $1,73 \mathrm{~m}^{2}$, e de 90 até $130 \mathrm{ml} / \mathrm{min}$, em mulheres adultas para uma superfície corporal de $1,73 \mathrm{~m}^{2}$.

$\mathrm{Na}$ análise dos dados laboratoriais, em relação ao tempo de observação, antes do óbito, considerou-se o seguinte registro seqüencial: 1) um a quatro meses antes do óbito; 2) dez a trinta dias antes do óbito; 3) cinco a dez dias antes do óbito; 4) dois a cinco dias antes do óbito; 5) zero a 48 horas antes do óbito.

Das amostras de rim fixadas em formol ou metacarn foram confeccionadas lâminas coradas pela hematoxilina-eosina, tricrômico de Masson, prata metenamina e picro-sirius, sendo analisadas ao microscópio de luz ${ }^{17}$. Quando necessário, foram realizadas técnicas histoquímicas para identificação de agentes infecciosos. Foram avaliados os quatro compartimentos do parênquima renal: glomerular, tubular, intersticial e vascular ${ }^{19}$.

O diagnóstico da infecção pelo VIH foi realizado através de dois testes de ELISA positivos e confirmado pelo Western blot ou imunofluorescência. As doenças definidoras de SIDA foram estabelecidas segundo os critérios do Centro para Doenças Infecciosas de Atlanta ${ }^{20}$.

Para comparar as freqüências, utilizou-se o $\chi^{2}$, sendo considerada a diferença estatisticamente significativa quando a probabilidade de rejeição da hipótese de nulidade foi menor que $5 \%$ $(\mathrm{p}<0,05)$. 


\section{RESULTADOS}

Características gerais da população estudada. Dos 119 pacientes, 99 (83\%) eram do sexo masculino. Quanto à cor, $70(59 \%)$ eram brancos, $33(28 \%)$ pardos e $16(13 \%)$ pretos. Em relação à conduta sexual, havia $42(35 \%)$ homossexuais, $59(50 \%)$ heterossexuais e $18(15 \%)$ ignorada. Entre eles, 51 (43\%) eram usuários de drogas endovenosas. A média das idades foi de $33 \pm 10,4$ anos, variando de 18 a 67 anos. Havia $91(76 \%)$ pacientes com idade compreendida entre 20 e 40 anos, sendo que 59 (50\%) eram menores de 30 anos. Observou-se que em 36 (30\%) pacientes, o diagnóstico sorológico foi feito na última internação, em um período menor que 30 dias antes do óbito, em 32 (27\%), entre 1 e 5 meses antes do óbito e em 51 (43\%), acima de 5 meses. As doenças definidoras do diagnóstico de SIDA mais freqüentes foram candidíase, toxoplasmose, pneumocistose e criptococose. Quanto aos diagnósticos anatomopatológicos, a NTI foi o mais freqüente (56\%), seguido de NTA $(36,1 \%)$ (Tabela 1$)$.

Tabela 1 - Diagnósticos anatomopatológicos renais em 119 necropsias de pacientes com SIDA.

\begin{tabular}{lcc}
\hline Diagnóstico & $\mathrm{N}^{\circ}$ & $\%$ \\
\hline Nefrite túbulo-intersticial & 67 & 56,0 \\
Necrose tubular aguda & 43 & 36,1 \\
Hialinose arteriolar & 27 & 22,7 \\
Nefrocalcinose & 18 & 15,1 \\
Tumores & 4 & 3,4 \\
Vasculopatia hipertensiva & 2 & 1,7 \\
GESF & 2 & 1,7 \\
Hiperplasia mesangial & 1 & 0,8 \\
Sem alterações & 17 & 14,6
\end{tabular}

Obs: O total ultrapassa $100 \%$ porque em alguns pacientes foram encontrados mais de um diagnóstico.

Manifestações clínicas e diagnósticos anatomopatológicos. Nos 119 indivíduos estudados, somente $22(18 \%)$ apresentaram edema de membros inferiores, de + ou $++/++++$. Destes, $10(45 \%)$ casos tinham creatininemia acima de $1,2 \mathrm{mg} / \mathrm{dl}$ nos 5 dias antes do óbito e 3 deles apresentaram valores acima de $3 \mathrm{mg} / \mathrm{dl}$ e diagnóstico anatomopatológico de NTA. Nos 12 restantes, que apresentaram edema, a creatininemia estava dentro dos limites normais e a histopatologia mostrou NTI em 5, NTA em 2 e normal em 5.

Nos 97 pacientes sem edema de membros inferiores, $33(32 \%)$ deles tinham creatinina acima de 1,2 e em 64 (65\%)a creatinina era normal, não tendo diferença estatisticamente significativa nestes dois grupos $(p=0,83)$.

Somente $2(1,6 \%)$ pacientes apresentaram valores de $150 \mathrm{mmHg}$ de tensão arterial sistólica; em 117 pacientes, a tensão era normal ou baixa, especialmente próximo ao óbito.

Dados laboratoriais e diagnósticos anatomopatológicos. Em 115 pacientes foram feitas 301 dosagens de creatinina sérica, sendo que 28 tiveram somente uma dosagem realizada.
Verificou-se que $63(55 \%)$ pacientes mantiveram o nível sérico de creatinina dentro dos limites normais em todos os períodos de avaliação. Em $15(13 \%)$, os níveis eram compatíveis com insuficiência renal estabelecida, ou seja, creatinina maior que $3 \mathrm{mg} / \mathrm{dl}$ e em $37(32 \%)$, a elevação da creatinina era menor que $3 \mathrm{mg} / \mathrm{dl}$. Considerando os níveis de creatinina em até 10 dias antes do óbito, período com maior probabilidade de ter correlação com o exame anatomopatológico, verificou-se que $45(39 \%)$ pacientes apresentaram elevação da creatininemia no período mencionado, sendo que em 22 a elevação da creatinina sérica foi discreta (Tabela 2).

Em 117 pacientes, foram feitas 294 dosagens de sódio e potássio. Em 38 (32\%) pacientes, não foram encontradas alterações nos níveis de sódio sérico. Em 71 (61\%) pacientes, houve pelo menos um episódio de hiponatremia, sendo que 19 pacientes se apresentaram hiponatrêmicos nas 48 horas antes do óbito. Em 66 (56\%) doentes, os episódios não foram graves e apareceram em diferentes períodos antes da morte, sendo que em 2 surgiram pelo menos um 
Tabela 2 - Diagnósticos anatomopatológicos de 45 pacientes com SIDA e creatinina sérica acima do valor normal nos 10 dias antes do óbito.

\begin{tabular}{lccccccccc}
\hline & & \multicolumn{7}{c}{ Diagnósticos anatomopatológicos } \\
\cline { 3 - 10 } Creatinina mg/dl & № & NTA & NTI & NTI +NTA & GESF + NTA & normal & NR & autólise grave & CA \\
\hline $1,3-1,9$ & 22 & 4 & 8 & 6 & 1 & 2 & 1 & & 1 \\
$2,0-3,0$ & 9 & 2 & 6 & & & & & 1 & 1 \\
$>3,0$ & 14 & 5 & 3 & 3 & 1 & & 1 & 1 \\
\hline Total & 45 & 11 & 17 & 9 & 2 & 2 & 2 & 1 & 1
\end{tabular}

№ = número de pacientes; NTA = necrose tubular aguda, NTI = nefrite túbulo-intersticial, GESF = glomeruloesclerose segmentar focal, $\mathrm{NR}=$ amostra não representativa, $\mathrm{CA}=$ carcinoma.

mês antes. Em 5 (4\%) pacientes, tais episódios foram graves, com sódio menor que $120 \mathrm{mEq} / \mathrm{l}$, ocorrendo dentro das 48 horas antes do óbito, em um deles e entre cinco e dez dias antes do óbito, nos outros 4 . Em 28 (24\%) pacientes, houve pelo menos um episódio de hipocalemia, sendo em 8 deles, dentro das 48 horas que antecedeu à morte; o menor valor encontrado foi de $1,5 \mathrm{mEq} / \mathrm{l}$.

Creatininemia e diagnósticos anatomopatológicos. Dos 45 casos com elevação da creatinina, os diagnósticos anatomopatológicos foram diversos (Tabela 2). Destes, 22 pacientes com creatinina $<2 \mathrm{mg} / \mathrm{dl}$, a NTA foi observada em 11 (50\%) e a NTI em 14 $(63,6 \%)$. Dos 9 com creatinina entre $2 \mathrm{mg} / \mathrm{dl}$ e $3 \mathrm{mg} / \mathrm{dl}$, encontrou-se $2(23 \%)$ casos com NTA e $6(73 \%)$ com NTI. Dos 14 com creatininemia > 3mg/ dl, observou-se $9(64 \%)$ com NTA e $6(42,9 \%)$ com NTI.

Por outro lado, quando se analisou os 43 pacientes com o diagnóstico de NTA observouse que $22(51 \%)$ tiveram alterações da creatininemia. Dos 67 casos de NTI, 26 (38,8\%) tiveram alterações da creatininemia.

Hiponatremia, hipocalemia e diagnósticos anatomopatológicos. Em $8(7 \%)$ pacientes havia hipernatremia e em 71 (61\%) hiponatremia. Nos 71 pacientes com hiponatremia, havia $45(63 \%)$ com NTI. Destes, em $32(71,1 \%)$ foram evidenciados agentes infecciosos: $13(40,6 \%)$ bactérias (7 Mycobacterium sp e 6 não classificada); $12(37,5 \%)$ fungos (6 Cryptococcus sp e 6 Histoplasma sp); 4 (12,5\%) vírus (citomegalovirus - CMV) e $3(9,3 \%)$ mistas (Cryptococcus sp + Mycobacterium sp, Histoplasma $s p+$ bactéria não classificada e Histoplasma sp + Candida sp). Nos outros 13 casos de NTI, em que não se identificaram agentes infecciosos, um caso era NTI xantogranulomatosa. Por outro lado, dos 67 pacientes com NTI, $45(67,2 \%)$ apresentaram hiponatremia.

Em 15 (36\%) casos com hiponatremia encontrou-se NTA. Em 7\% pacientes havia hipercalemia e em 28 (24\%) hipocalemia. Dos 28 pacientes que apresentaram hipocalemia, houve $16(57 \%)$ pacientes com diagnóstico anatomopatológico de NTI, 5 com NTA e 5 tiveram NTI e NTA.

Depuração de creatinina, proteinúria de 24 horas e diagnósticos anatomopatológicos. Em 12 dos 24 pacientes, nos quais foi calculada a depuração de creatinina e a proteinúria de 24 horas, os níveis de proteínas na urina estavam elevados. Somente um deles tinha proteinúria de nível nefrótico, o qual apresentava glomeruloesclerose segmentar focal (GESF). Em 7 encontrou-se mais de $1 \mathrm{~g}$, em algum momento do estudo, e em 5 as dosagens estiveram entre $150 \mathrm{mg}$ e $1 \mathrm{~g}$ (Tabela 3).

Pacientes sem alteração anatomopatológica. Em 17 (14\%) pacientes não foram encontradas alterações renais ao exame anatomopatológico. Em um deles, a creatinina estava pouco aumentada $(1,3 \mathrm{mg} / \mathrm{dl})$ no dia do óbito, sendo que os valores anteriores estavam dentro dos limites normais. Outro apresentou hipernatremia nos 10 dias antes do óbito $(159 \mathrm{mEq} / \mathrm{l})$. Houve hiponatremia em 6 pacientes, nos 10 dias antes do óbito, sendo o menor valor de $126 \mathrm{mEq} / \mathrm{l}$. Dois apresentaram hipocalemia, respectivamente de 2,9 e $2,3 \mathrm{mEq} / \mathrm{l}$. 
Tabela 3 - Depuração de creatinina, proteinúria de 24 horas e diagnósticos anatomopatológicos dos pacientes com SIDA.

\begin{tabular}{lcccl}
\hline Caso & $\begin{array}{c}\text { Proteinúria 24h } \\
\text { máxima - mg (seqüencial) }\end{array}$ & $\begin{array}{c}\text { Creatinina máxima } \\
\mathrm{mg} / \mathrm{dl} \text { (seqüencial) }\end{array}$ & $\begin{array}{c}\text { Depuração de creatinina } \\
\text { (seqüencial) }\end{array}$ & \multicolumn{1}{c}{ Rim - anatomopatológico } \\
\hline 1 & $5500(3)$ & $1,5(4)$ & $106(3)$ & GESF; NTA; hialinose \\
2 & $2137(5)$ & $2,3(5)$ & $20(5)$ & NTI Cryptococcus sp; NTA \\
3 & $1968(2)$ & $0,6(3)$ & $121(3)$ & NTA; NTI por citomegalovírus \\
4 & $1868(4)$ & $3,4(4)$ & $19(4)$ & NTA;NTI inespecífica \\
5 & $1512(2)$ & $1,1(2)$ & $91(2)$ & NTI por Mycobacterium sp \\
6 & $1276(1)$ & $7,0(5)$ & $35(1)$ & GESF; NTA \\
7 & $1238(5)$ & $1,5(2)$ & $51(2)$ & NTA \\
8 & $742(2)$ & $0,9(3)$ & $108(3)$ & NTI por Cryptococcus $s p$ \\
9 & $605(1)$ & $1,2(4)$ & $16(1)$ & Amostra insuficiente \\
10 & $336(1)$ & $1,6(4)$ & $12(4)$ & NTI por Cryptococcus $s p ;$ hialinose \\
11 & $323(1)$ & $1,8(5)$ & $5(5)$ & NTA \\
12 & $188(5)$ & $1,3(5)$ & NTA insuficiente
\end{tabular}

(seqüencial) = tempo antes do óbito: (1) - um a quatro meses, (2) - dez a trinta dias, (3) - cinco a dez dias (4) - dois a cinco dias, (5) zero a 48 horas.

\section{DISCUSSÃO}

Quanto às manifestações clínicas, encontrouse $22(18 \%)$ pacientes com discreto edema de membros inferiores. Os pacientes com SIDA, mesmo apresentando hipoproteinemia e hipercatabolismo, habitualmente não se apresentam com edema importante ${ }^{47}$. Apenas 3 pacientes com edema apresentavam também disfunção renal com níveis elevados de creatinina e no exame histopatológico do rim apresentavam NTA, provavelmente uma coincidência, pois a NTA isolada não seria causa do edema. Comparando-se este grupo com aqueles que apresentavam edema sem elevação da creatinina, não houve diferença estatisticamente significativa.

No presente estudo, considerou-se o valor da creatinina sérica de $3 \mathrm{mg} / \mathrm{dl}$ como indicativo de insuficiência renal estabelecida (IRE), para maior possibilidade de correlação anatomopatológica. O valor de creatinina sérica que define a insuficiência renal, varia de acordo com alguns autores: $1,2 \mathrm{mg} / \mathrm{dl}^{22}, 2 \mathrm{mg} / \mathrm{d}^{35}$, enquanto outros não utilizam valores absolutos, mas sim a variação num dado período de tempo em relação à creatinina basal ${ }^{32}$. Dos 115 casos com dosagem de creatinina, encontrou-se elevação em 45 (39\%) casos em contraste com o $55 \%$ descritos ${ }^{35}$. Desses, $14(12,2 \%)$ apresentavam-se com IRE, prevalência menor que o $20 \%$ descrita pelo mesmo autor ${ }^{35}$.

Podemos observar, ainda, que quando encontrada elevação da creatininemia, a correspondência anatomopatológica nem sempre estava presente. Dos 45 casos com elevação da creatinina, em $49 \%$ deles encontrouse NTA, e dos 14 casos com IRE, a NTA estava presente em $64 \%$. A literatura mostra causas variadas de NTA em indivíduos com SIDA ${ }^{326} 35$. Em um estudo de 146 casos de IRA/NTA, a isquemia foi a causa em $52 \%$ dos casos, seguida por drogas nefrotóxicas em $23 \%$. A sepse foi responsável direta ou indiretamente por $75 \%$ das IRAs nestes pacientes ${ }^{25}$. Em outro estudo, a depleção de volume foi responsável por 38\% de elevação de creatinina, sendo que a taxa de mortalidade próxima de $70 \%$ na NTA com inestabilidade hemodinâmica ${ }^{35}$. A NTA encontrada no presente estudo, pode ser devido tanto à isquemia, quanto à toxicidade por drogas. Em nossa amostra, os pacientes com NTA não apresentaram com freqüência infecção do parênquima renal de modo que não possamos relacioná-las. Os pacientes com SIDA freqüentemente são hospitalizados com infecções múltiplas, complicações gastrointestinais e outras doenças, necessitando de tratamento com drogas nefrotóxicas todos os quais são fatores de risco para o desenvolvimento de NTA ${ }^{326}$. A maioria destes pacientes está hemodinamicamente instável, devido à sobreposição de hipovolemia, hiponatremia, acidose metabólica e respiratória com comprometimento cardiorrespiratório ${ }^{326}$.

Dos 45 casos com elevação da creatinina, em $58 \%$ encontrou-se NTI e dos 14 com IRE, em $43 \%$ havia NTI, sendo que em $50 \%$ estava associado a NTA. Encontrou-se também NTI 
xantogranulomatosa associada a IRE, com alteração da arquitetura histológica, observandose grande quantidade de macrófagos com lipídeos no seu interior caracterizando as células xantomatosas.

A NTI alérgica por drogas tem sido relatada de 9 a 13,3\%835. Um caso de NTI com IRE apresentou infiltrado inflamatório mononuclear difuso juntamente com eosinófilos, fato não encontrado nos outros casos. Achamos que poderia este caso estar relacionado ao uso de drogas toda vez que o paciente fez uso de cotrimoxazol, oxacilina, ceftriaxona e antiinflamatórios não-esteróides, podendo ser o mecanismo alérgico a causa da $\mathrm{NTI}^{19}$.

As NTI poderiam também ocasionar insuficiência renal. Em alguns casos de NTI havia destruição do parênquima e grande quantidade de agente infeccioso. Em 3 dos 16 pacientes com Cryptococcus sp no rim, constatou-se elevação da creatinina e alteração na sua depuração. Na histopatologia, observou-se numerosas colônias junto a lesões histolíticas extensas e pouca reação inflamatória freqüentemente focal, mostrando a pouca capacidade de resposta, decorrente do estado imunológico do paciente.

Contudo, alguns pacientes com NTA e/ou NTI apresentaram creatinina sérica normal. A melhor correlação seria com a depuração de creatinina. No entanto, os poucos dados não nos permitem avaliar que percentagens de casos de NTA e/ou NTI poderiam apresentar-se com depuração de creatinina normal. Os casos com creatinina normal e NTA no diagnóstico anatomopatológico, podem ser devido ao tempo transcorrido entre a obtenção da amostra sangüínea e o óbito. Muitas vezes a NTA é um evento terminal, próximo ao óbito, acometendo pacientes com septicemia ou falência de múltiplos órgãos ${ }^{26}$. Além disso, podese encontrar quadros de NTA focal, em que nem todos os néfrons são acometidos de forma homogênea, permitindo manter a função do órgão dentro dos limites normais, devido a reserva funcional do rim $^{6}$.

Em relação aos distúrbios hidroeletrolíticos, a alta freqüência de pacientes com hiponatremia (61\%) concorda com outros relatos, sendo o distúrbio hidroeletrolítico mais freqüente entre os pacientes com SIDA 1312222834 . As percentagens descritas variam de 40 a $52 \%$ dos casos ${ }^{1102234}$; estas diferenças devem-se a alguns fatores, entre eles o valor utilizado para definir hiponatremia, a caracterização clínica dos pacientes, e os exames realizados para classificar as suas diferentes causas. Múltiplas causas condicionam a hiponatremia, tais como: diarréia, desidratação, insuficiência renal, infecções pulmonares, acometimento do sistema nervoso central, como encefalites ou atrofia cortical. Estas duas últimas podem ocasionar síndrome de secreção inadequada de hormônio antidiurético (SIHAD) 3101229.

Por outro lado, encontrou-se $7 \%$ de hipernatremia, em comparação com 24 a 75\% dos pacientes hospitalizados apresentados por outro autor $^{10}$. Este distúrbio hidroeletrolítico pode resultar das perdas insensíveis na febre ou deficiência na capacidade renal para concentrar a urina devido às infecções, ou ao tratamento com rifampicina, anfotericina $\mathrm{B}^{2} \mathrm{e}$ foscarnet ${ }^{22}$. Em relação aos distúrbios do potássio, a hipocalemia aparece como conseqüência das perdas gastrointestinais, por vômito e diarréia, do uso prolongado de anfotericina $B$, ou da administração de foscarnet ${ }^{2}$. Com menor freqüência, os pacientes com SIDA apresentam-se com hipercalemia relacionada à insuficiência supra-renal ${ }^{13}$, ao uso de cotrimoxazol ${ }^{11}$ ou pentamidina ${ }^{15}$.

Não tem sido descrita, alteração morfológica que se associe diretamente a hiponatremia. Encontrou-se hiponatremia em $63 \%$ dos casos, associados a NTI, e dos 5 pacientes que tiveram hiponatremia grave, em 4 houve associação com NTI. Em um deles ocorreu dentro das 48 horas antes do óbito, sendo grande a possibilidade de ter contribuído para o mesmo. A hiponatremia freqüentemente está relacionada a distúrbios funcionais. No presente estudo, além dos prováveis distúrbios funcionais, é possível que a NTI possa ter colaborado ou facilitado o aparecimento da hiponatremia. Sabe-se que cerca de $99 \%$ do sódio é reabsorvido quando termina de passar pelos túbulos distais e as lesões tubulares poderiam ter impedido o normal processo de reabsorção. O Cryptococcus sp foi o agente infeccioso mais freqüentemente encontrado no rim. No entanto, não se encontrou relação entre desequilíbrio hidro-eletrolítico e criptococose.

As NTI, freqüentemente observadas em pacientes com o HIV, podem ser decorrentes não só do uso de drogas nefrotóxicas, como também devido aos diferentes agentes infecciosos, incluindo o próprio HIV. As interrelações entre o HIV e células tubulares têm sido estudadas ${ }^{1428}$, além das relações entre leucócitos infectados com o HIV e células tubulares renais, estas 
parecem produzir interleucinas (IL-6 e TNF $\alpha$ ) que estimulam a expressão de HIV nos monócitos infectados que infiltram o rim e devem desempenhar algum papel na gênese da lesão renal ${ }^{24}$. Assim mesmo, já foi sugerido que o TGF $\beta$ está envolvido na patogênese da nefropatia associada ao HIV e o bloqueio deste fator poderia ser importante no tratamento dessa nefropatia ${ }^{37}$.

As alterações glomerulares foram as primeiras a serem descritas em pacientes com SIDA ${ }^{92527}$, sendo encontrado GESF em até 10\% e hipercelularidade mesangial de 25 a $35 \%$. No entanto, a freqüência destas alterações glomerulares, parece variar de acordo com a região e a raça ${ }^{31}{ }^{36}$. Na presente casuística, na maioria dos casos, as alterações glomerulares primárias estavam ausentes ou eram discretas, semelhante a alguns trabalhos ${ }^{831}$. Encontrou-se apenas $1,7 \%$ dos casos com GESF, semelhante a outros estudos que relatam de $1,1 \%$ a $2 \%{ }^{21} 31$. Um dos pacientes com GESF apresentava clinicamente azotemia, proteinúria de nível nefrótico sem edemas periféricos. O outro paciente apresentava proteinúria não nefrótica, porém, a dosagem foi feita com muita antecedência ao óbito, podendo não refletir o quadro final do paciente. Este paciente evoluiu rapidamente com IRE e faleceu, sendo encontrado também NTA.

Foram encontrados vinte e sete casos com hialinose arteriolar e $60 \%$ deles tinham menos de 40 anos $^{17}$, será necessário caracterizar melhor, em estudos futuros, este acometimento vascular nos pacientes com SIDA; já que é sabido que a hialinose arteriolar se apresenta geralmente em pacientes com diabetes melitus, com hipertensão arterial ou em pessoas de idade avançada, a diferença da hialinose detectada neste trabalho, que predominou em pacientes jovens.

Os achados anatomopatológicos renais no exame pós-morte, da presente amostra, apresentaram uma ampla variedade de alterações clínicas, dados semelhantes aos descritos na literatura ${ }^{8}$. As alterações renais em indivíduos necropsiados, difere das provenientes de biópsias renais ${ }^{3} 9$. Aquelas freqüentemente exibem alterações intersticiais, pois os pacientes ficam expostos a vários agentes infecciosos, podendo evoluir para sepse terminal, além do uso de drogas potencialmente nefrotóxicas ${ }^{3} 8$. Nas amostras provenientes de biópsias, os indivíduos apresentam uma manifestação renal, sendo maior a chance de se encontrar glomerulopatias ${ }^{3}$. Além disso, parece que a freqüência e a natureza das complicações renais nos pacientes com SIDA variam consideravelmente de uma região a outra ${ }^{31}$.

Concluiu-se que as alterações clínicolaboratoriais nos pacientes com SIDA são variadas, bem como as alterações anatomopatológicas renais. As alterações glomerulares foram relativamente raras e as lesões túbulo-intersticiais foram muito freqüentes, principalmente a NTI e a NTA. Nem sempre podese observar uma correlação anátomo-clínica, pois muitas vezes a alteração foi predominantemente funcional, especialmente nas alterações hidroeletrolíticas.

\section{AGRADECIMENTOS}

Os autores agradecem ao Prof. Omar da Rosa Santos, pelas valiosas sugestões e críticas a este trabalho.

\section{REFERÊNCIAS BIBLIOGRÁFICAS}

1. Agarwal A, Soni A, Ciechanowsky M, Chander P, Treser G. Hyponatremia in patients with the acquired imunodeficiency syndrome. Nephron 53:317-321, 1989.

2. Berns JS, Cohen RM, Stumacher RJ, Rudnick MR. Renal aspects of therapy for human immunodeficiency virus and associated opportunistic infections. Journal of the American Society of Nephrology 1:1061-1080, 1991.

3. Bourgoignie JJ. Renal complications of human immunodeficiency virus type 1 . Kidney international 37:1571-1584, 1990.

4. Bourgoignie JJ, Meneses R, Ortiz C, Jaffe D, Pardo V. Clinical spectrum of renal disease associated with human immunodeficiency virus. American Journal of Kidney Diseases 12:131-137, 1988.

5. Brady HR, Brenner BM. Acute renal failure. In: Isselbacher KJ, Braunwald E, Martin JB, Fauci AS, Kasper DL, Wilson JD (eds) Harrison's principles of internal medicine. $13^{\text {th }}$ edition. New York, p. 1265-1273, 1994.

6. Brenner BM, Herbert SC. Disturbances of renal function. In: Isselbacher KJ, Braunwald E, Martin JB, Fauci AS, Kasper DL, Wilson JD (eds) Harrison's principles of internal medicine. $13^{\text {th }}$ edition. New York, p. 1259-1264, 1994. 
7. Cohen AH, Nast CC. HIV-associated nephropathy: a unique combined glomerular, tubular and interstitial lesion. Modern Pathology 1:87-97,1988.

8. D'Agati V, Cheng JI, Carbone L, Cheng JT, Cappel G. The pathology of HIV-nephropathy. A detailed morphologic and comparative study. Kidney international 35:1358-1370, 1989.

9. Gardenswartz MH, Lerner CW, Seligson GR, Zabetakis PM, Rotterdam H, Tappez ML, Michelis MF, Bruno MS. Renal disease in patients with AIDS: a clinicopathologic study. Clinical Nephrology 21:197-204, 1984.

10. Glassock R, Ucla Conference. Human immunodeficiency virus (HIV). Infection and the kidney. Annals of Internal Medicine 112:35-49, 1990.

11. Greenberg S, Reiser IW, Chou S-Y, Porush JG. Trimethoprim-sulfamethoxazole induces reversible hyperkalemia. Annals of Internal Medicine 119:291-295, 1993.

12. Hofbauer LC, Heufelder, AE Endocrine implications of human immunodeficiency virus infection. Medicine 75:262-278, 1996.

13. Kalin Mf, Poretsky L, Seres Ds, Zumoff B. Hyporeninemic hypoaldosteronism associated with acquired immunodeficiency syndrome. The American Journal of Medicine 82:1035-1038, 1987.

14. Kapasi A, Bhat P, Singhal PC. Tubular cell and HIV-1 gp120 interaction products promote migration of monocytes. Inflammation 22:137-144, 1998.

15. Lachaal M, Venuto RC. Nephotoxicity and hyperkalemia in patients with acquired immunodeficiency sindrome treated with pentamidine. The American Journal of Medicine 87: 260-263, 1989.

16. Lafayette RA, Perrone RD, Levey AS. Laboratory evoluation of renal function. In: Schrier RW, Gottschalk CW (eds) Diseases of the kidney. $6^{\text {th }}$ edition. New York, p. 307-354, 1996.

17. Laguna-Torres VA. Alterações renais nos pacientes com SIDA e sua expressão clínico-laboratorial e anatomopatológica. Tese de mestrado. Universidade de Brasília, Brasília DF, 1997.

18. Laguna-Torres VA, Reis MA, Menegaz RA, Pelá GA, Prata A. Alterações anatomopatológicas renais em indivíduos com a síndrome da imunodeficiência adquirida. Revista da Sociedade Brasileira de Medicina Tropical 31:465-472, 1998.

19. Lajoie G, Silva FG. Approach to the interpretation of renal biopsy. In: Silva FG, D’Agati VD, Nadasdy T (eds) Renal biopsy interpretation. Churchill Livingstone, New York, p. 31-70,1996.

20. Leads from the MMWR. Revision of the CDC surveillance case definition for acquired immunodeficiency syndrome. JAMA 258:1143-1153, 1987.

21. Lopes GS, Marques LPG, Rioja L, Basilio-De-Oliveira CA, Oliveira AV, Nery ACF, Santos OR. Glomerular disease and human immunodeficiency virus infection in Brazil. American Journal of Nephrology 12:281-287, 1992.

22. Manfro RC, Stumpf AG, Horn CL, Wolffenbuttel I, Harsein RC, Prompt CC, Kronfeld M, Kopstein J. Alterações hidroeletrolíticas, ácido-base, e da função renal em portadores de SIDA. Revista da Associação Médica Brasileira 39:43-48, 1993.

23. Navarro JF, Quereda C, Gallego N, Antera A, Mora C, Ortuno J. Nephrogenic diabetes insipidus and renal tubular acidosis secondary to foscarnet therapy. American Journal of Kidney Diseases 27:431-434, 1996.

24. O'Donnell MP, Chao CC, Gekker G, Modi KS, Kasiske $\mathrm{BL}, \mathrm{Keane} W \mathrm{~F}$. Renal cell cytokine production stimulates HIV-1 expression in chronically HIV-1-infected monocytes. Kidney International 53:593-597, 1998.

25. Rao TKS, Filipone EJ, Nicastri AA, Landesman SH, Frank E, Chen CK, Friedman EA. Associated focal and segmental glomeruloesclerosis in the acquired immunodeficiency syndrome. The New England Journal of Medicine 310:669-673, 1984.

26. Rao TKS, Friedman EA. Outcome of severe acute renal failure in patients with acquired immunodeficiency syndrome. American Journal of Kidney Diseases 25:390398, 1995.

27. Rao TKS, Friedman EA, Nicastri AD. The types of renal disease in the acquired immunodeficiency syndrome. The New England Journal of Medicine 316:1062-1068, 1987.

28. Ray PE, Liu XH, Henry D, Dye III L, Xu L, Orenstein JM, Schuztbank TE. Infection of human primary renal epithelial cells with HIV-1 from children with HIVasscociated nephropathy. Kidney International 53:12171229, 1998.

29. Santos Gil I, Polo G, Del Arco C, Martinez MC, Noguerado A. Síndrome de secreción inadecuada de ADH asociado a síndrome de inmunodeficiencia adquirida. Revista Clinica Española 188:120-122, 1991.

30. Santos OR, Lopes GS. Renal complications of AIDS. A latin american perspective. A current Survey of the world literature 3:63-70, 1994.

31. Seney FC, Burns DK, Silva FG. Acquired immunodeficiency syndrome and the kidney. American Journal of Kidney Diseases 16:1-13, 1990.

32. Shusterman N, Strom BL, Murray TG, Morrison G,West $S$ L, Maislin G. Risk factor and outcome of hospitalacquired acute renal failure clinical epidemiologic study. The American Journal of Medicine 83:65-76, 1987.

33. Slot C. Plasma creatininte determination: a new and specific Jaffé reaction method. Scandinavian Journal of Clinical and Laboratory Investigation 17:381-387, 1965.

34. Tang WW, Kaptein EM, Feinstein E, Massry SG. Hyponatremia in hospitalized patients with the acquired immunodeficiency syndrome (AIDS) and the AIDS-related complex. The American Journal of Medicine 94:169-174, 1993. 
35. Valeri A, Neusy AJ. Acute and chronic renal disease in hospitalizated AIDS patients. Clinical Nephrology 35:110118, 1991.

36. Winston J, Klotman PE. HIV-associated nephropathy. The Mount Sinai Journal of Medicine 65:27-32, 1998.
37. Yamamoto $T$, Noble N, Miller DE. Increased levels of transfering growth factor $\mathrm{b}$ in HIV-associated nephropathy. Kidney International 55:579-592, 1999. 